\title{
PROPRIEDADES DA MADEIRA DE Pinus caribaea E Eucalyptus grandis ESTIMADAS POR COLORIMETRIA
}

\author{
Patrícia Gomes Ribeiro Amorim*, Joaquim Carlos Gonçalez², José Arlete Alves Camargos ${ }^{3}$
}

*Autora para correspondência: ribeiropg@unb.br

RESUMO: A utilização de tecnologias alternativas não destrutivas tem se mostrado bastante eficiente na caracterização e na avaliação da qualidade da madeira. Neste estudo, objetivou-se caracterizar tecnologicamente as madeiras de Pinus caribaea e Eucalyptus grandis por meio da Colorimetria. O estudo foi desenvolvido na Universidade de Brasília (UnB), no Laboratório de Engenharia e Física do Laboratório de Produtos Florestais/LPF - Serviço Florestal Brasileiro (SFB). As espécies foram submetidas a ensaios convencionais de densidade básica, retratibilidade e flexão estática. Para a determinação dos parâmetros colorimétricos, utilizou-se um espectrofotômetro, conforme metodologia adotada por Camargos (1999). A face radial da madeira é a mais adequada à coleta dos parâmetros colorimétricos e predição das propriedades. A propriedade melhor estimada por essa técnica foi a densidade, seguida pelo módulo de elasticidade (MOE) e módulo de ruptura (MOR).

Palavras-chave: Parâmetros colorimétricos, propriedades físicas e mecânicas, correlações.

\section{WOOD PROPERTIES OF Eucalyptus grandis AND Pinus caribaea ESTIMATED BY COLORIMETRY}

\begin{abstract}
The use of non-destructive technologies has been very effective in characterization and evaluation of wood quality. This study aimed to characterize the technologically wood of Pinus caribaea and Eucalyptus grandis by Colorimetry. The study was conducted at the University of Brasilia (UNB) in Physics and Engineering Laboratory the Laboratory of Forest Products/ $L P F$ - Brazilian Forest Service (SFB). Species were subjected to standard tests of basic density, shrinkage and bending. For the colorimetric determination of the parameters it was used a spectrophotometer according to the methodology adopted by Camargos (1999). The radial surface of the wood is most suitable for the collecting of the colorimetric parameters and prediction of properties. Property best estimated by this technique was the density, followed by modulus of elasticity (MOE) and modulus of rupture (MOR).
\end{abstract}

Key words: Colorimetric parameters, physical and mechanical properties, correlations.

\section{INTRODUÇÃO}

O desenvolvimento de novas tecnologias, e o aprimoramento de técnicas tradicionais é o modo mais eficiente de melhorar a qualidade dos produtos fabricados pela indústria e utilizar de forma racional o potencial madeireiro disponível nas florestas. Para melhorar a qualidade dos produtos, é necessário o conhecimento prévio das propriedades da madeira a ser utilizada, podendo com isso maximizar seu uso e minimizar os problemas do processo produtivo. A utilização de metodologias eficientes, rápidas e "não destrutivas" contribui para alcançar esses objetivos.

As técnicas não destrutivas são ferramentas utilizadas na caracterização da madeira e na avaliação da durabilidade, apresentam vantagens em relação às tecnologias tradicionais, pois é possível avaliar uma peça sem ter de extrair dela corpos de prova, um grande volume de material pode ser

${ }^{1}$ Universidade Federal do Acre - Rio Branco, Acre, Brasil

${ }^{2}$ Universidade de Brasília - Brasília, Distrito Federal, Brasil

${ }^{3}$ Serviço Florestal Brasileiro - Brasília, Distrito Federal, Brasil analisado com maior rapidez e precisão, se torna versátil para se enquadrar numa rotina de linha de produção, podendo ser empregado a nível industrial, no segmento madeira e produtos derivados, agregando valores, melhorando a qualidade e a eficiência dos produtos fabricados.

Dentre estas técnicas enquadram-se a avaliação visual (colorimetria e análise de imagens), química (infravermelho próximo) e por ondas de tensão e ultrassônicas (stress wave timer e ultrassom).

A cor da madeira é influenciada por diversos fatores, tais como: genética, anatomia, composição química, plano ortogonal da madeira (BURGER; RICHTER, 1991; GONÇALEZ, 1993; MONTES et al., 2008; PANDEY, 2005; RIBEIRO et al., 2005). A cor, entretanto, não é estável em uma madeira, uma vez que ela tende a alterarse com a ação do intemperismo, seja pela lixiviação ocasionada pela chuva ou ainda pela fotodegradação (PASTORE et al., 2008; ROSU et al., 2010).

Cerne, Lavras, v. 19, n. 3, p. 461-466, jul./set. 2013 
Visando sempre ao uso final, a determinação da cor se destaca como um fator fundamental para a caracterização da qualidade da madeira, que influirá diretamente no aspecto visual e, consequentemente, na sua comercialização (MOYA; MARIN, 2011). Tornase importante, pois permite uma classificação por cor com ajuda das coordenadas cromáticas, o que pode, por exemplo, melhorar o preço da madeira no mercado, além de tornar o produto mais homogêneo quanto ao seu desenho e textura, e se transformar em um critério relevante na hora da compra (GONÇALEZ et al., 2001; JANIN et al., 2001).

O sistema CIELAB (Comission International de L'Eclairage ou Comissão Internacional de Iluminantes), atualmente utilizado na determinação quantitativa da cor (colorimetria), é um método que define a sensação da cor, baseado em três elementos: a luminosidade $\left(\mathrm{L}^{*}\right)$, a tonalidade (coordenadas cromáticas a* e b*) e a saturação (C) (CAMARGOS, 1999).

A colorimetria vem evoluindo ano a ano, fazendo da propriedade cor um fator chave na qualidade da madeira, podendo ainda ser utilizada, para avaliar propriedades mecânicas (MOR e MOE) de painéis de partículas orientadas de madeira (THOMPSOM, 2006) e propriedades físicas e mecânicas de madeira maciça (RIBEIRO, 2009).

Nesse contexto, neste estudo, objetivouse determinar as propriedades físicas (densidade, retratibilidade e anisotropia) e mecânicas (flexão estática: módulos de elasticidade e ruptura - MOE e MOR) das madeiras de Pinus caribaea var. hondurensis e Eucalyptus grandis, utilizando a colorimetria (parâmetros colorimétricos) com base no sistema CIELAB 1976.

\section{MATERIAL E MÉTODOS}

Neste estudo, foram utilizadas duas espécies de madeiras: Pinus caribaea var. hondurensis e Eucalyptus grandis. A madeira de pinus foi doada por uma empresa do Distrito Federal, com idade estimada de 21 anos. A madeira de eucalipto foi proveniente de um plantio de 13 anos de idade, no Distrito Federal. De cada espécie, foram obtidas cinco pranchas. Desse material, foram confeccionados 12 corpos de prova, nas dimensões de $2 \mathrm{~cm} \times 2 \mathrm{~cm} \times 10 \mathrm{~cm}$ e 2 $\mathrm{cm} \times 2 \mathrm{~cm} \times 30 \mathrm{~cm}$, necessários aos ensaios convencionais (densidade e retratibilidade-COPANT 30:1 - 005/461/72 e 005/462/71; flexão estática - COPANT 30:1 - 006/72), além da colorimetria (COMISIÓN PANAMERICANA DE NORMAS TÉCNICAS - COPANT, 1972a, 1972b, 1972c).
Os ensaios foram realizados na Universidade de Brasília e no Laboratório de Engenharia e Física do Laboratório de Produtos Florestais do Serviço Florestal Brasileiro (LPF/SFB). Para o ensaio de colorimetria, foi utilizado um espectrofotômetro Datacolor Microflash 200d acoplado a um microcomputador, com iluminante D65 e ângulo de $10^{\circ} \mathrm{C}$, em temperatura ambiente. Foram realizadas 25 leituras para cada face (radial e tangencial), totalizando 50 leituras por corpo de prova para cada espécie.

A metodologia adotada para a determinação dos parâmetros colorimétricos foi o sistema CIELAB 1976, conforme Camargos (1999). Os parâmetros colorimétricos determinados foram $\mathrm{L}^{*}$ (luminosidade), coordenadas cromáticas a* e b*, C (saturação) e h* (ângulo de tinta).

A saturação (C) foi determinada pela Equação 1 e o ângulo de tinta pela Equação 2, conforme se segue:

$C=\left(a^{* 2}+b^{* 2}\right)^{1 / 2}$

$h^{*}=\operatorname{tg}^{-1} \frac{b^{*}}{a^{*}}$

onde: $\mathrm{C}=$ saturação; $\mathrm{a}^{*}=$ coordenada cromática sobre $\mathrm{o}$ eixo verde-vermelho; $b^{*}=$ coordenada cromática sobre $o$ eixo azul-amarelo; $h^{*}=$ ângulo de tinta.

Os resultados obtidos nos ensaios convencionais foram submetidos à análise estatística descritiva: cálculo de média, mínimo, máximo e desvio padrão. Os resultados obtidos nos ensaios convencionais foram correlacionados com os resultados do ensaio de colorimetria por Pearson a $1 \%$. Foram calculadas regressões para ajustes de equações, estimando valores de propriedades físicas e mecânicas das madeiras em estudo. Foram empregados a ANOVA (análise de variância), e Tukey a $5 \%$ de probabilidade.

\section{RESULTADOS, DISCUSSÃO}

Os valores das propriedades físicas e mecânicas das madeiras de pinus e eucalipto estão apresentados na Tabela 1.

Os valores médios dos parâmetros colorimétricos das madeiras de pinus e eucalipto são apresentados na Tabela 2 e comparados a outras espécies reportadas na literatura.

A cor da madeira de pinus é caracterizada pela luminosidade $\left(\mathrm{L}^{*}=56,87\right)$, pelas coordenadas a* $(4,66)$ e b* $(18,85)$, além do ângulo de tinta $\left(h^{*}=76,10\right)$ e saturação $(C=19,43)$. Segundo a classificação proposta por Camargos (1999), o pinus é uma madeira clara, pois possui valor de L* maior que 54, e de coloração branca amarelada.

Cerne, Lavras, v. 19, n. 3, p. 461-466, jul./set. 2013 
Tabela 1 - Valores médios para a densidade básica, retratibilidades (volumétrica, tangencial e radial; V, T e R, respectivamente), anisotropia e módulos de elasticidade (MOE) e ruptura (MOR) das madeiras de pinus e eucalipto.

Table 1 -Average values for density, shrinkage (volumetric, tangential and radial; $V$, $T$ and $R$, respectively), anisotropy and modulus of elasticity (MOE) and rupture (MOR) of pine and eucalyptus woods.

\begin{tabular}{lccccccc}
\hline \multirow{2}{*}{ Espécie } & $\rho$ & \multicolumn{3}{c}{ Retratibilidade $(\%)$} & & Anisotropia & \multicolumn{2}{c}{ Flexão Estática } \\
\cline { 3 - 7 } & $\left(\mathrm{g} / \mathrm{cm}^{3}\right)$ & $\mathrm{V}$ & $\mathrm{T}$ & $\mathrm{R}$ & Razão (T/R) & MOE $(\mathrm{Mpa})$ & $\mathrm{MOR}(\mathrm{Mpa})$ \\
\hline \multirow{3}{*}{ Pinus } & 0,46 & 12,47 & 8,21 & 4,91 & 1,83 & 10084,54 & 91,58 \\
& $(0,03)(0,07)$ & $(1,28)(0,10)$ & $(0,73)(0,09)$ & $(0,50)(0,10)$ & $(0,45)(0,24)$ & $(840,14)(0,08)$ & $(458,51)(0,04)$ \\
& $(0,42)(0,51)$ & $(10,33)(15,20)$ & $(7,06)(9,36)$ & $(4,09)(5,82)$ & $(1,25)(2,55)$ & & 104,26 \\
\multirow{3}{*}{ Eucalipto } & 0,56 & 13,76 & 7,65 & 6,25 & 1,22 & 10546,02 & 1 \\
& $(0,02)(0,03)$ & $(0,86)(0,06)$ & $(0,69)(0,09)$ & $(0,68)(0,11)$ & $(0,22)(0,18)$ & $(8,56)(0,09)$ & $(5,27)(0,05)$ \\
& $(0,54)(0,59)$ & $(12,69)(15,38)$ & $(6,64)(8,85)$ & $(5,10)(7,27)$ & $(0,86)(1,57)$ & &
\end{tabular}

Valores entre parêntesis são, respectivamente, desvio-padrão, coeficiente de variação (\%), valores mínimo e máximo.

Tabela 2 - Valores médios dos parâmetros colorimétricos $\left(\mathrm{L}^{*}\right.$, $\left.\mathrm{a}^{*}, \mathrm{~b}^{*}, \mathrm{C}, \mathrm{h}^{*}\right)$ das madeiras de pinus e eucalipto comparados a outras espécies.

Table 2 - Average values of colorimetric parameters $\left(L^{*}, a^{*}, b^{*}\right.$, $\left.C, h^{*}\right)$ of pine and eucalyptus wood compared to other species.

\begin{tabular}{lccccc}
\hline \multirow{2}{*}{ Espécie } & \multicolumn{5}{c}{ Parâmetros colorimétricos } \\
\cline { 2 - 6 } & $\mathrm{L}^{*}$ & $\mathrm{a}^{*}$ & $\mathrm{~b}^{*}$ & $\mathrm{C}$ & $\mathrm{h}^{*}$ \\
\hline Pinus & 56,87 & 4,66 & 18,85 & 19,43 & 76,16 \\
Eucalipto & 46,77 & 10,57 & 14,08 & 17,61 & 53,10 \\
Tauari $^{2}$ & 57,73 & 7,21 & 14,41 & 16,12 & 63,37 \\
Ipê $^{3}$ & 39,00 & 10,00 & 16,00 & 18,90 & 58,00 \\
Mogno $^{3}$ & 52,10 & 14,60 & 28,70 & 32,10 & 63,10 \\
Pau marfim $^{3}$ & 84,10 & 3,30 & 21,80 & 22,10 & 81,40 \\
Muirapiranga $^{1}$ & 42,39 & 22,02 & 15,56 & 26,99 & 35,10 \\
Hevea $^{1}$ & 77,55 & 6,56 & 19,61 & 20,74 & 71,74 \\
\hline
\end{tabular}

${ }^{1}$ Autran e Gonçalez (2006); ${ }^{2}$ Ribeiro et al. (2005); ${ }^{3}$ Gonçalez et al. (2001).

A coordenada $b^{*}$ (pigmentação amarela) é a principal responsável pela formação da cor desta madeira. Entretanto, a pigmentação vermelha (coordenada $\mathrm{a}^{*}$ ) se faz presente influenciando a composição da coloração da espécie. $\mathrm{O}$ ângulo de tinta $\left(\mathrm{h}^{*}\right)$ confirma a maior proximidade do eixo amarelo, evidenciando o tom amarelado da madeira. Comparando com outras espécies madeireiras (Tabela 2), verifica-se a particularidade da cor da espécie estudada. A madeira dessa espécie tem uma maior proximidade com as madeiras de pau marfim e seringueira, levando em consideração a luminosidade e o ângulo de tinta. Assim, o seu uso em ambientes interiores poderá agregar valor estético, dando uma opção interessante para a indústria.
A cor da madeira de eucalipto é caracterizada pela luminosidade $\left(\mathrm{L}^{*}=46,77\right)$, pelas coordenadas a* $(10,57) \mathrm{e}$ $b^{*}(14,08)$, além do ângulo de tinta $\left(h^{*}=53,10\right)$ e saturação $(C=17,61)$. Segundo Camargos (1999), o eucalipto é uma madeira escura ( $L^{*}<$ que 54$)$ e de coloração rosa forte. A coordenada a* (pigmentação vermelha) é a principal responsável pela formação da cor dessa madeira. Entretanto, a pigmentação amarela (coordenada $b^{*}$ ) se faz presente influenciando a composição da coloração dessa espécie. $\mathrm{O}$ ângulo de tinta $(\mathrm{h} *)$ confirma a maior proximidade do eixo vermelho, evidenciando a importância do pigmento vermelho $\left(\mathrm{a}^{*}\right)$.

Os parâmetros colorimétricos da madeira de pinus e eucalipto para as faces tangencial e radial são apresentados na Tabela 3.

Tabela 3 - Valores médios, das faces radial e tangencial, dos parâmetros colorimétricos $\left(\mathrm{L}^{*}, \mathrm{a}^{*}, \mathrm{~b}^{*}, \mathrm{C}, \mathrm{h}^{*}\right)$ das madeiras de pinus e eucalipto.

Table 3 - Average values of radial and tangential faces, the colorimetric parameters $\left(L^{*}, a^{*}, b^{*}, C^{*} h\right)$ of the wood of pine and eucalyptus woods.

\begin{tabular}{lcccccc}
\hline \multirow{2}{*}{ Espécie } & \multirow{2}{*}{ Faces } & \multicolumn{5}{c}{ Parâmetros colorimétricos } \\
\cline { 3 - 7 } & & $\mathrm{L}^{*}$ & $\mathrm{a}^{*}$ & $\mathrm{~b}^{*}$ & $\mathrm{C}$ & $\mathrm{h}^{*}$ \\
\hline \multirow{2}{*}{ Pinus } & Radial & $56,53^{\mathrm{a}}$ & $4,72^{\mathrm{a}}$ & $18,86^{\mathrm{a}}$ & $19,45^{\mathrm{a}}$ & $75,98^{\mathrm{a}}$ \\
& Tangencial & $57,21^{\mathrm{b}}$ & $4,60^{\mathrm{b}}$ & $18,84^{\mathrm{a}}$ & $19,40^{\mathrm{b}}$ & $76,45^{\mathrm{b}}$ \\
\hline \multirow{2}{*}{ Eucalipto } & Radial & $47,22^{\mathrm{a}}$ & $10,63^{\mathrm{a}}$ & $14,25^{\mathrm{a}}$ & $17,78^{\mathrm{a}}$ & $53,28^{\mathrm{a}}$ \\
& Tangencial & $46,33^{\mathrm{b}}$ & $10,50^{\mathrm{b}}$ & $13,91^{\mathrm{b}}$ & $17,44^{\mathrm{b}}$ & $52,92^{\mathrm{b}}$ \\
\hline
\end{tabular}

Valores seguidos pela mesma letra, dentro da mesma espécie e na mesma coluna, são considerados estatisticamente iguais a 5\% de probabilidade pelo teste de Tukey.

Cerne, Lavras, v. 19, n. 3, p. 461-466, jul./set. 2013 
Analisando os valores dos parâmetros colorimétricos obtidos para as madeiras de pinus e eucalipto, verificou-se que existem diferenças significativas entre as duas faces da madeira a $5 \%$ de probabilidade pelo teste de Tukey. Na madeira de pinus, a face radial é mais escura ( $L^{*}$ é menor e o a* é maior) que a tangencial. Enquanto na madeira de eucalipto tem-se o inverso, já afirmado por Gonçalez et al. (2006) ao estudar as características tecnológicas das madeiras de E. grandis e E. cloeziana. A coordenada $\mathrm{a}^{*}$ (coloração vermelha) é a principal responsável pela coloração mais escura desta face.

A análise dos parâmetros colorimétricos da madeira, por ser quantitativa, permite estimar suas propriedades físicas e mecânicas. Nas Figuras de 1 a 3, mostra-se a separação nítida das espécies estudadas em dois grupos, relacionando a densidade e os parâmetros colorimétricos.

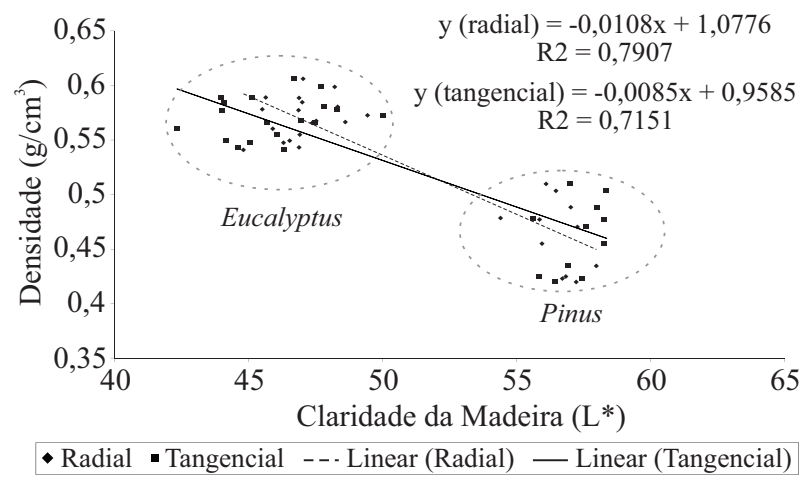

Figura 1 - Análise de regressão da densidade das madeiras de pinus e eucalipto em função da luminosidade da madeira ( $\left.\mathrm{L}^{*}\right)$.

Figure 1-Regression analysis of density of pine and eucalyptus wood according to the luminosity $\left(L^{*}\right)$.

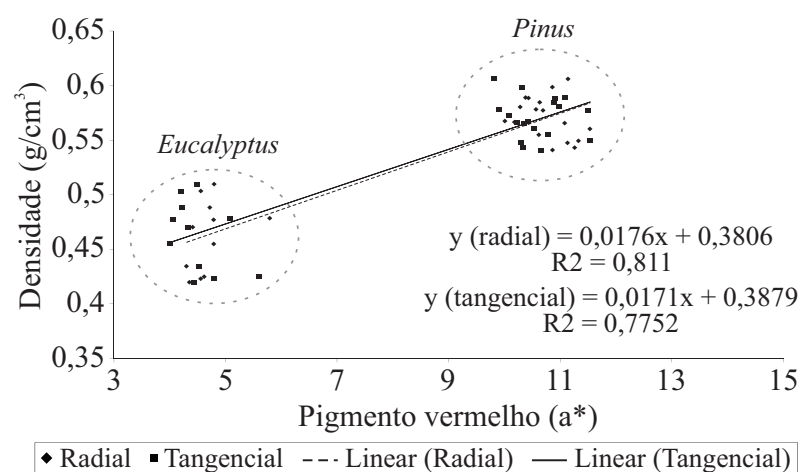

Figura 2 -Análise de regressão da densidade das madeiras de pinus e eucalipto em função do pigmento vermelho da madeira (a*).

Figure 2 - Regression analysis of wood density of pine and eucalyptus according to the red pigment ( $a *)$.

Cerne, Lavras, v. 19, n. 3, p. 461-466, jul./set. 2013

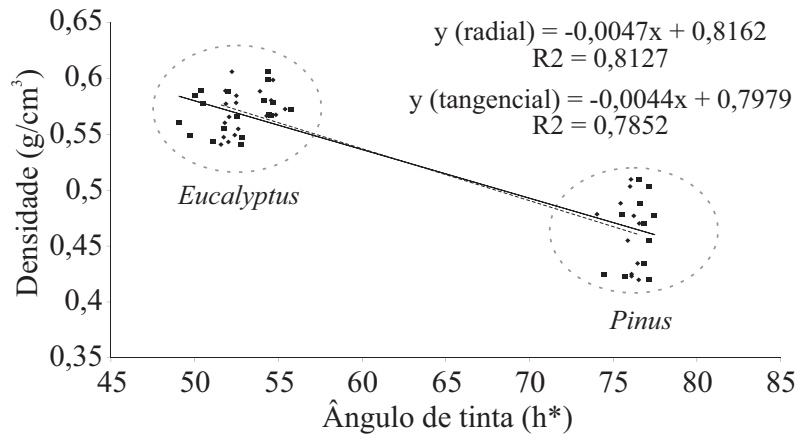

- Radial - Tangencial --- Linear (Radial) — Linear (Tangencial)

Figura 3 - Análise de regressão da densidade das madeiras de pinus e eucalipto em função do ângulo de tinta $\left(\mathrm{h}^{*}\right)$.

Figure 3 - Regression analysis of wood density of pine and eucalyptus according to the angle of ink $\left(h^{*}\right)$.

As correlações entre $\mathrm{L}^{*}, \mathrm{a}^{*}, \mathrm{~b}^{*}, \mathrm{C}$ e $\mathrm{h}^{*}$ e as propriedades de densidade, anisotropia e MOR para as madeiras de pinus e eucalipto mostraram-se altas e promissoras $(-0,70$ a 0,$76 ;-0,64$ a 0,$68 ;-0,61$ a 0,53$)$. Ao correlacionar essas variáveis, observa-se que a densidade e o MOR apresentam o mesmo padrão de correlação, com valores de coeficientes de correlação maiores para a densidade (Tabela 4).

Tabela 4 - Correlação entre os parâmetros colorimétricos ( $\mathrm{L}^{*}$, $\left.a^{*}, b^{*}, C, h^{*}\right)$ e propriedades físicas e mecânicas da madeira de pinus e eucalipto.

Table 4-Correlation between colorimetric parameters ( $L * a *$, $\left.b^{*}, C^{*} h\right)$ and physical and mechanical properties of pine and eucalyptus woods.

\begin{tabular}{lccccc}
\hline \multirow{2}{*}{ Propriedades } & \multicolumn{5}{c}{ Correlações } \\
\cline { 2 - 6 } & $\mathrm{L}^{*}$ & $\mathrm{a}^{*}$ & $\mathrm{~b}^{*}$ & $\mathrm{C}$ & $\mathrm{h}^{*}$ \\
\hline $\begin{array}{l}\text { Densidade } \\
\text { Retratibilidade }\end{array}$ & $-0,70$ & 0,76 & $-0,77$ & $-0,75$ & $-0,76$ \\
volumétrica & $-0,16$ & 0,13 & $-0,08$ & 0,01 & $-0,12$ \\
Anisotropia & 0,61 & $-0,64$ & 0,68 & 0,67 & 0,66 \\
MOE & $-0,04$ & 0,01 & $-0,05$ & $-0,07$ & $-0,01$ \\
MOR & $-0,59$ & 0,53 & $-0,60$ & $-0,61$ & $-0,56$ \\
\hline
\end{tabular}

Analisando a densidade observa-se correlação negativa para todos os parâmetros exceto para o a*. Observa-se que quanto maior o valor de $\mathrm{L}^{*}$, menor a densidade da madeira e quanto maior o valor de $\mathrm{a}^{*}$ maior a densidade (Figuras 1 e 2). Macedo (2002), estudando $E$. grandis e $E$. cloeziana, afirma que a pigmentação vermelha 
tende a aumentar, à medida que a espessura da parede celular aumenta (XAVIER, 2008), e pode ser comprovado pela correlação positiva da coordenada a* com a espessura da parede celular das fibras.

A correlação entre a densidade e o valor médio do ângulo de tinta ( $\mathrm{h}^{*}$ ) foi o que apresentou maior coeficiente de determinação $\left(R^{2}=0,82\right)$, sendo o menor $R^{2}(0,67)$ representado pela relação densidade $\mathrm{X}$ saturação $(\mathrm{C})$. O melhor modelo para sua estimativa foi em relação ao ângulo de tinta $\left(\mathrm{h}^{*}\right)$, onde: . Nesse caso, é possível prever até $82 \%$ da propriedade com um erro de $4 \%$.

Observando-se as Figuras 1 e 2, verifica-se que a face radial representada pelos parâmetros colorimétricos $L^{*}$ e a* são os mais recomendados para estimar a densidade.

Comparando a face radial e tangencial da madeira (Figura 3), a radial é a que melhor prevê os valores de densidade, quando considerados os parâmetros: luminosidade, pigmentação vermelho e ângulo de tinta.

Apesar do coeficiente de anisotropia e do módulo de ruptura (MOR) das duas espécies mostrarem correlação significativa com os parâmetros colorimétricos $\mathrm{L}^{*} \mathrm{e} \mathrm{b}^{*}$, os valores de $\mathrm{R}^{2}$ foram baixos (menores que 0,46 ), sugerindo que novas pesquisas devem ser desenvolvidas, aumentando o número de amostras e repetições para confirmar essas evidências.

Não foi encontrada correlação significativa entre a retratibilidade volumétrica e os parâmetros colorimétricos. Assim como entre os parâmetros colorimétricos e o módulo de elasticidade (MOE) da madeira de pinus e eucalipto. Já, para o módulo de ruptura (MOR) as correlações foram significativas a $1 \%$.

Para o módulo de ruptura (MOR), apesar de todos os coeficientes de determinação $\left(\mathrm{R}^{2}\right)$ serem baixos, as análises foram significativas e o melhor modelo para sua estimativa foi em relação a luminosidade da madeira, permitindo uma estimativa de $40 \%$ o valor do MOR, onde: $Y=0,2079 x^{2}-22,78 x+712,76$.

O erro padrão dá uma boa precisão da estimativa quando apresentam valores baixos. Esses erros para as relações com a densidade básica estão na ordem de 5\%. As demais relações apresentam erros acima de 30\%.

\section{CONCLUSÕES}

A colorimetria quantitativa, como técnica para a determinação de propriedades físico-mecânicas da madeira, mostrou-se adequada. Por meio dos parâmetros colorimétricos fornecidos pelo sistema CIELAB 1976, foi possível diferenciar as madeiras de Pinus caribaea var. hondurensis e Eucalyptus grandis e estimar as suas propriedades.

\section{REFERÊNCIAS}

AUTRAN, C. S.; GONÇALEZ, J. C. Caracterização colorimétrica das madeiras de muirapiranga (Brosimum rubescens Taub.) e de seringueira (Hevea brasiliensis, clone Tjir 16 Müll Arg.) visando à utilização em interiores. Revista Ciência

Florestal, Santa Maria, v. 16, n. 4, p. 445-451, jul./ago. 2006.

BURGER, L. M.; RICHTER, H. G. Anatomia da madeira. São Paulo: Nobel, 1991. 154 p.

CAMARGOS, J. A. A. Colorimetria aplicada na elaboração de uma tabela de cores para madeiras tropicais. 1999. 99 p. Dissertação (Mestrado em Ciências Florestais) - Universidade de Brasília, Brasília, 1999.

COMISIÓN PANAMERICANA DE NORMAS TÉCNICAS. COPANT 30: 1-006: maderas: método de determinación de flexión estática. La Paz, 1972a.

COMISIÓN PANAMERICANA DE NORMAS TÉCNICAS.

COPANT 461: maderas: método de determinación del peso especifico aparente. La Paz, 1972 b.

\section{COMISIÓN PANAMERICANA DE NORMAS TÉCNICAS.}

COPANT 462: maderas: método de determinación de contracción. La Paz, 1972c.

GONÇALEZ, J. C. Caracterization technologique de quatre especies peu connues de la forêt amazonienne: anatomie, chimie, couleur, propriétés physiques et mécaniques. 1993. $446 \mathrm{p}$. Thèse (Doctorat en Technologie du Bois) - Ministere de l'Agriculture et de la Peche, Nancy, 1993.

GONÇALEZ, J. C.; BREDA, L. de C. S.; BARROS, J. F. M.; MACEDO, D. G.; JANIN, G.; COSTA, A. F. da; VALE, A. T. do. Características tecnológicas das madeiras de Eucalyptus grandis W.Hill ex Maiden E Eucalyptus cloeziana F. Muell visando ao seu aproveitamento na indústria moveleira. Revista Ciência Florestal, Santa Maria, v. 16, n. 3, p. 329-341, 2006.

GONÇALEZ, J. C.; JANIN, G.; SANTORO, A. C. S.; COSTA, A. F. da; VALE, A. T. do. Colorimetria quantitativa: uma técnica objetiva de determinar a cor da madeira. Brasil Florestal, Rio de Janeiro, n. 72, p. 47-58, 2001.

Cerne, Lavras, v. 19, n. 3, p. 461-466, jul./set. 2013 
JANIN, G.; GONÇALEZ, J. C.; ANANIAS, R.; CHARRIER, B.; SILVA, G. F.; DILEM, A. Aesthetics appreciation of wood colour and pattern s by colorimetry: part 1, colorimetry theory for the CIELAB system. Maderas: Ciencia y Tecnología, San José, v. 3, n. 1/2, p. 3-13, 2001.

MACEDO, D. G. Aspecto mercadológico da madeira de Eucalyptus grandis e Eucalyptus cloeziana para a indústria moveleira. 2002. 57 p. Dissertação (Mestrado em Ciências Florestais) - Universidade de Brasília, Brasília, 2002.

MONTES, C. S.; HERNANDEZ, R. E.; BEAULIEU, J.; WEBER, J. Genetic variation in wood color and its correlations with tree growth and wood density of Calycophyllum spruceanum at an early age in the Peruvian Amazon. New Forests, Dordrecht, v. 35, p. 57-73, 2008.

MOYA, R.; MARIN, J. D. Grouping of Tectona grandis (L.f.) clones using wood color and stiffness. New Forests, Dordrecht, v. 42, p. 329-345, 2011.

PANDEY, K. K. Study of the effect of photo-irradiation on the surface chemistry of wood. Polymer Degradation and Stability, Essex, v. 90, p. 9-20, 2005.

PASTORE, T. C. M.; OLIVEIRA, C. C. K. de; RUBIM, J. C.; SANTOS, K. O. Efeito do intemperismo artificial em quatro madeiras tropicais monitorado por espectroscopia de infravermelho (DRIFT). Química Nova, São Paulo, v. 31, n. 8, p. 2071-2075, 2008.
RIBEIRO, P. G. Utilização de técnicas não destrutivas para caracterização de madeiras de Pinus caribaea Var. hondurensis e de Eucalyptus grandis. 2009. 114 p. Dissertação (Mestrado em Ciências Florestais) - Universidade de Brasília, Brasília, 2009.

RIBEIRO, P. G.; GONÇALEZ, J. C.; CAMARGOS, J. A. A. de; MARTINS, I. S. Avaliação colorimétrica das faces radial e tangencial da madeira de tauari (Allantoma lineata) visando sua utilização em interiores. In: REUNIÃO ANUAL DA SBPC, 57., 2005, Fortaleza. Anais... Fortaleza: SBPC, 2005. 1 CD-ROM.

ROSU, D.; TEACA, C. A.; BODIRLAU, R.; ROSU, L. FTIR and color change of the modified wood as a result of artificial light irradiation. Journal of Photochemistry and Photobiology B: Biology, Lausanne, v. 99, p. 144-149, 2010.

THOMPSOM, R. M. Resistência de painéis de partículas orientadas (OSB) tratados termicamente frente ao intemperismo. 2006. 48 p. Monografia (Graduação em Engenharia Florestal) - Universidade de Brasília, Brasília, 2006.

XAVIER, R. B. L. Avaliação da dureza Janka, densidade e estabilidade de quatro espécies de Eucalyptus implantadas no Estado do Rio de Janeiro. 2008. 31 p. Monografia (Graduação em Engenharia Florestal) - Universidade Federal Rural do Rio de Janeiro, Seropédica, 2008.

Recebido: 29 de dezembro de 2010; aceito: 28 de fevereiro de 2013.

Cerne, Lavras, v. 19, n. 3, p. 461-466, jul./set. 2013 